Gazi University
Journal of Science
http://dergipark.gov.tr/gujs

\title{
Some Statistics in Polar Coordinate System with Uniform Angles
}

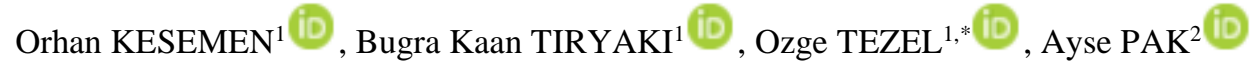 \\ ${ }^{I}$ Department of Statistics and Computer Sciences, Faculty of Science, Karadeniz Technical University, Trabzon, Turkey \\ ${ }^{2}$ Department of Medical Informatics/Bioinformatics, Faculty of Medicine, Karadeniz Technical University, Trabzon, Turkey
}

\author{
Highlights \\ - The paper focuses on some statistics in polar coordinate system with uniform angles. \\ - The proposed method defines the probability density functions in polar coordinates. \\ - The proposed method generates random numbers in polar coordinates. \\ - The proposed method performs goodness of fit test in polar coordinates. \\ - The validity of the proposed method is shown in the simulation study.
}

\section{Article Info}

Received: $23 / 08 / 2019$

Accepted: 09/04/2020

Keywords

Goodness of fit test

Polar distribution

Polar histogram

Random number

\begin{abstract}
In this study, some bivariate distribution functions are defined in the polar coordinate system and random numbers are generated from these distribution functions. In these definitions, the angular change of the probability density function is taken as constant, and the distance change is performed based on the univariate probability density function. Also, the chi-square goodnessof-fit test is proposed for random numbers generated in the polar coordinates. Four different distribution functions are selected to evaluate the success of the proposed chi-square goodness of fit test for polar distribution functions. Lastly, the validity and the success of the proposed method is shown in the simulation study and the real-life example.
\end{abstract}

\section{INTRODUCTION}

Distribution function constitutes the fundamental basis of the statistical theory. About the distribution function, the first thing comes to mind is the univariate distribution functions. In the case of two variables, the joint distribution function of two variables is used. These joint distribution functions cause problems such as the independence condition and the inverse of the functions. Therefore, the marginal and the conditional distribution functions are preferred instead of the joint distribution functions. The random variable $X$, represented by each distribution function, is defined as the continuous random variable or the discrete random variable according to the content of the domain. In this study, only continuous distribution functions are used.

Bivariate distribution functions are generally defined in the cartesian coordinates. However, in some applications, they are also defined in the polar coordinates. In these applications, making the definitions with polar variables will provide conveniences both in terms of the representation and the solving of the problem. The determination of the position of ships in the marine, the object detection by radar in the defense industry, the motion of the robot arm in the robot technology, the target setting of anti-aircraft, the calculation of the statistics of telephone and television transmitters and the investigation of the effect of a factory emitting toxic gas into the air can be exemplified as these applications.

Although there are not many statistical studies in polar coordinates, there are different studies in the literature using polar probability density function and polar representations. Some of these studies are; Alford et. al. performed a modeling by using polar probability density function [1]. Solman and Kingstone 
introduced a novel head-contingent display system with using polar probability density function [2]. Schönefeld converted probability distributions from polar to Cartesian coordinates [3]. Cumming and DeAngelis compared two animal species using polar probability density function [4]. Robinson et.al. used a polar probability density function inherent in the polar geometry [5]. Schomaker et. al. superposed the polar diagrams of the edge-direction distribution [6]. Fazio et. al. used the distribution functions for the polar angle $\theta$ [7].

The polar coordinate system is a two-dimensional system. This system is used in many fields such as mathematics, biology, physics, engineering, marine, robotics, anti-aircraft, unmanned aerial vehicle (uav), telephone transmitters. The polar coordinate system is used when the relationship between two coordinates is more easily expressed in terms of the angle and the distance. This relationship is also determined by trigonometric formulas. In some real-life problems, it is more advantageous to use polar coordinate system instead of cartesian coordinates. Especially, the polar coordinate system is used frequently in the abovementioned applications. Contributing to the solutions of real-life problems in polar coordinates and creating solutions to such applications provide the motivation of this study. Especially, the motivation of this study comes from the fact that contrary to the papers in the literature, the proposed method defines the probability density functions in polar coordinates, generates random number from these distributions and performs goodness of fit test in polar coordinates.

Some statistics in polar coordinate system with uniform angles is performed in this study. Some polar distributions are presented in Section 2. Histogram in the polar coordinates with the equal interval radius and with the equal slice areas are given in Section 3. In Section 4, random number generation from polar distributions are presented. The chi-square goodness of fit test for polar distributions is proposed in Section 5. The validity and the success of the proposed method is shown in Section 6. In Section 7, a real-life application of this study is given for showing the implementation of the polar distributions.

\section{POLAR DISTRIBUTIONS}

The usage of the bivariate probability functions in the probability theory increases gradually [8]. Although the usage of the bivariate distribution functions in the Cartesian coordinates is much more, the conversion of them to the polar coordinate system will facilitate the solution of the problems in many fields. The polar coordinates are used in the polar distribution functions. These coordinates are the distance $(r)$ to the starting point and the angle $(\theta)$ with the horizontal axis. Bivariate cumulative distribution function used in Cartesian coordinates is defined as,

$$
F_{X, Y}(x, y)=\int_{-\infty}^{y} \int_{-\infty}^{x} f_{X, Y}(x, y) d x d y
$$

and the polar cumulative distribution function is given in the following equation $[9,10]$

$$
(\theta, r)=\int_{0}^{\theta} \int_{0}^{r} f_{\Theta, \mathrm{R}}(\phi, \rho) \rho d \rho d \phi
$$

If the equivalence in the Equation (3) is correct,

$$
f_{X, Y}(x, y) \equiv f_{\Theta, \mathrm{R}}(\theta, r) .
$$

The Equation (4) will be used as the smallest calculation unit [11].

$d x d y=r d r d \theta$

Four different distribution functions are determined in the polar coordinates. These distributions are polar uniform distribution, polar exponential distribution, polar normal distribution and polar triangular distribution. 


\subsection{Polar Uniform Distribution}

When the angular change is taken constant, the probability density function of uniform distribution in polar coordinates is calculated as follows [12]

$$
f_{\Theta, \mathrm{R}}(\theta, r ; a, b)=\left\{\begin{array}{cc}
\frac{1}{\pi\left(b^{2}-a^{2}\right)}, & a<r<b \\
0, & \text { otherwise }
\end{array}\right.
$$

The graph of this probability density function with parameters $a=1$ and $b=2$ is given in Figure 1(a). The distribution function dependent on the variable $r$ is defined in the Equation (6),

$$
F_{\Theta, \mathrm{R}}(\theta, r ; a, b)=\int_{0}^{\theta} \int_{a}^{r} f_{\Theta, \mathrm{R}}(\theta, r ; a, b) r d r d \theta,
$$

and if the integral of this function is calculated, the Equation (7) is obtained

$$
F_{\Theta, \mathrm{R}}(\theta, r ; a, b)=\theta \int_{a}^{r} \frac{1}{\pi\left(b^{2}-a^{2}\right)} r d r
$$

The cumulative distribution function in the Equation (8) is obtained by calculating integral with bounds

$F_{\Theta, \mathrm{R}}(\theta, r ; a, b)=\frac{\theta}{2 \pi}\left[\frac{r^{2}-a^{2}}{b^{2}-a^{2}}\right]$.

The representations of the probability density and the cumulative distribution functions of the polar uniform distribution with parameters $a=1$ and $b=2$ are shown in the Figure 1 .

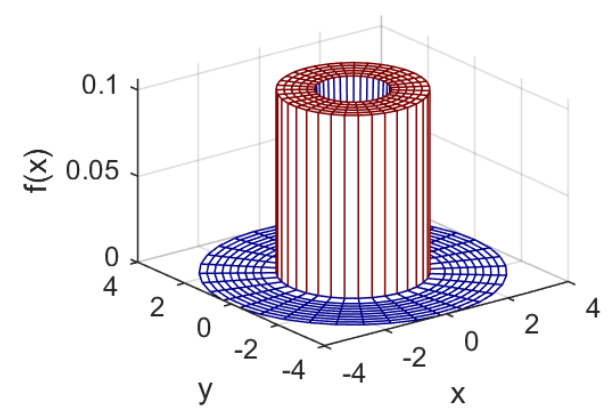

(a)

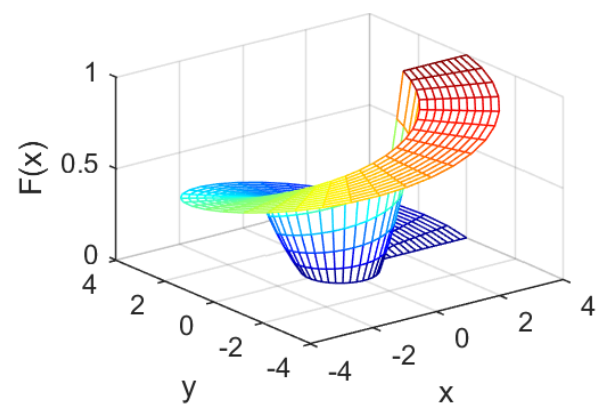

(b)

Figure 1. The polar uniform distribution with parameters $a=1$ and $b=2 ;(a)$ the probability density function $(b)$ the cumulative distribution function

The marginal probability density function dependent on the $R$ of the polar uniform distribution is calculated by using the Equation (9) as follows

$f_{R}(r ; a, b)=\int_{0}^{2 \pi} \frac{1}{\pi\left(b^{2}-a^{2}\right)} r d \theta$.

The marginal probability density function dependent on the $R$ of the polar uniform distribution is obtained by calculating integral with bounds as the following Equation (10)

$f_{R}(r ; a, b)=\frac{2 r}{\left(b^{2}-a^{2}\right)}$ 
The graph of the marginal probability density function dependent on the $R$ of the polar uniform distribution with parameters $a=1$ and $b=2$ is given in the Figure 2(a). If the integral of Equation (10) with bounds $[a, r]$ dependent on the $r$ is calculated, the Equation (11) is obtained

$$
F_{\mathrm{R}}(r ; a, b)=\int_{a}^{r} \frac{2 \rho}{\left(b^{2}-a^{2}\right)} d \rho
$$

Thus, the marginal cumulative distribution function of the polar uniform distribution dependent on $R$ is found as follows [12]

$$
F_{R}(r ; a, b)=\frac{r^{2}-a^{2}}{b^{2}-a^{2}}
$$

The graphs of the marginal probability density and the marginal cumulative distribution functions of the polar uniform distribution dependent on $R$ with parameters $a=1$ and $b=2$ are shown in the Figure 2 .

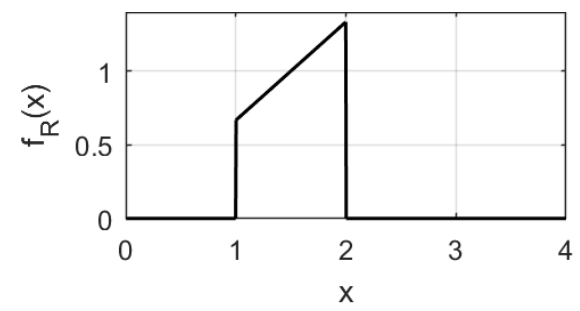

(a)

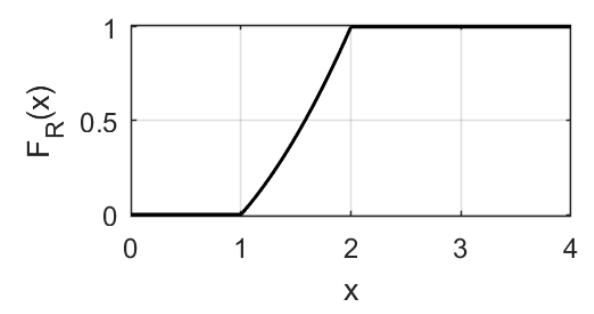

(b)

Figure 2. The polar uniform distribution dependent on $R$ with parameters $a=1$ and $b=2$; (a) the marginal probability density function $(b)$ the marginal cumulative distribution function

The marginal probability density function dependent on the $\Theta$ of the polar uniform distribution is calculated by using the Equation (5) as follows [12]

$f_{\Theta}(\theta ; a, b)=\int_{a}^{b} \frac{1}{\pi\left(b^{2}-a^{2}\right)} r d r$.

The marginal probability density function dependent on the $\Theta$ of the polar uniform distribution is obtained by calculating integral with bounds as the following Equation (14)

$f_{\Theta}(\theta ; a, b)=\frac{1}{2 \pi}$.

If the integral of Equation (14) with bounds $[0, \theta]$ dependent on the $\theta$ is calculated, the Equation (15) is obtained

$F_{\Theta}(\theta ; a, b)=\int_{0}^{\theta} \frac{1}{2 \pi} d \phi$

Thus, the marginal cumulative distribution function of the polar uniform distribution dependent on $\Theta$ is found as follows

$$
F_{\Theta}(\theta ; a, b)=\frac{\theta}{2 \pi}
$$

The graphs of the marginal probability density and the marginal cumulative distribution functions of the polar uniform distribution dependent on $\Theta$ are shown in the Figure 3. 


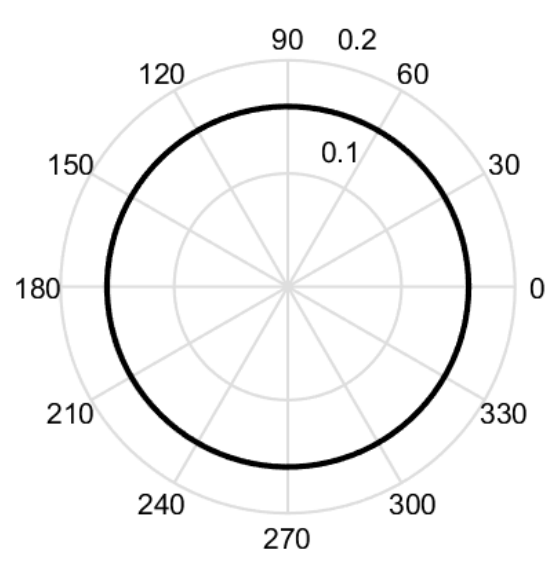

(a)

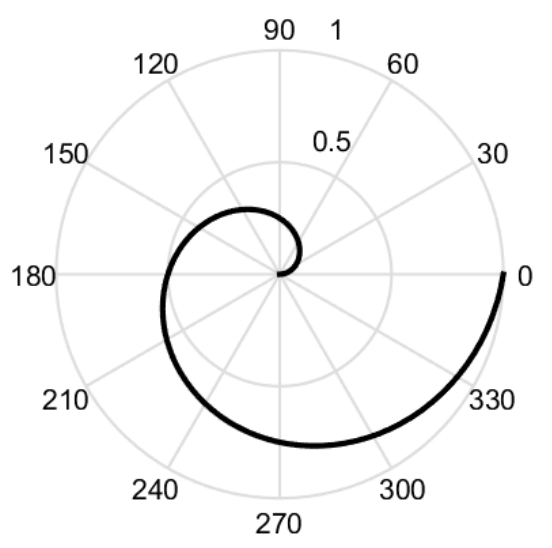

(b)

Figure 3. The polar uniform distribution dependent on $\Theta$ with parameters $a=1$ and $b=2$; (a) the marginal probability density function $(b)$ the marginal cumulative distribution function

\subsection{Polar Exponential Distribution}

When the angular change is taken constant, the probability density function of exponential distribution in polar coordinates is calculated as follows [12]

$f_{\Theta, \mathrm{R}}(\theta, r ; \lambda)=\frac{\lambda^{2} e^{-\lambda r}}{2 \pi}$

The graph of this probability density function with parameter $\lambda=2$ is given in Figure 4 (a). The distribution function dependent on the variable $r$ is defined in the Equation (18),

$$
F_{\Theta, \mathrm{R}}(\theta, r ; \sigma)=\int_{0}^{\theta} \int_{0}^{r} f_{\Theta, \mathrm{R}}(\phi, \rho ; \sigma) d \rho d \phi
$$

and if the integral of this function is calculated, the Equation (19) is obtained

$F_{\Theta, \mathrm{R}}(\theta, r ; \lambda)=\frac{\theta}{2 \pi} \int_{0}^{r} \lambda^{2} e^{-\lambda \rho} \rho d \rho$

The cumulative distribution function in the Equation (20) is obtained by calculating integral with bounds

$F_{\Theta, \mathrm{R}}(\theta, r ; \lambda)=\frac{\theta}{2 \pi}\left[1-(1+\lambda r) e^{-\lambda r}\right]$

The representations of the probability density and the cumulative distribution functions of the polar exponential distribution with parameter $\lambda=2$ are shown in the Figure 4.

The marginal probability density function dependent on the $R$ of the polar exponential distribution is calculated by using the Equation (17) as follows [12]

$f_{R}(r ; \lambda)=\int_{0}^{2 \pi} \frac{\lambda^{2} e^{-\lambda r}}{2 \pi} r d \phi$ 


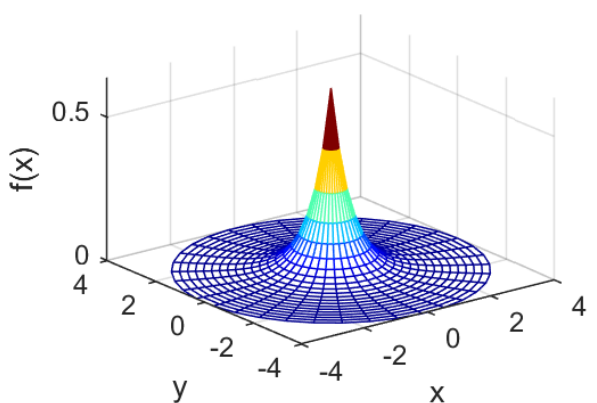

(a)

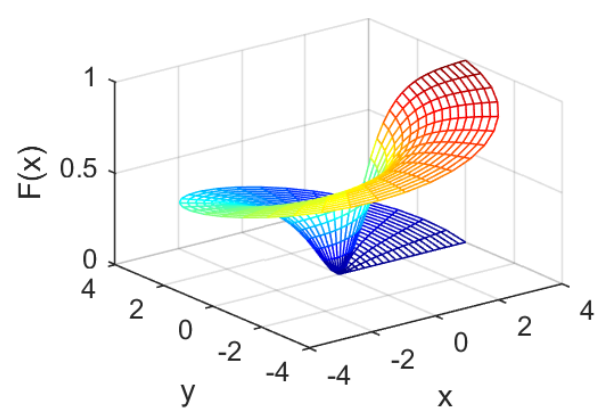

(b)

Figure 4. The polar exponential distribution with parameter $\lambda=2$; (a) the probability density function (b) the cumulative distribution function

The probability density function of the polar exponential distribution is obtained by calculating integral with bounds as follows

$f_{R}(r ; \lambda)=\lambda^{2} r e^{-\lambda r}$.

The graph of the marginal probability density function dependent on the $R$ of the polar exponential distribution with parameter $\lambda=2$ is given in the Figure 5(a). If the integral of Equation (22) with bounds $[0, r]$ dependent on the $r$ is calculated, the Equation (23) is obtained

$F_{\mathrm{R}}(r ; \lambda)=\int_{0}^{r} \lambda^{2} \rho e^{-\lambda \rho} d \rho$.

Thus, the marginal cumulative distribution function of the polar exponential distribution dependent on $R$ is found as follows

$$
F_{R}(r ; \lambda)=1-(1+\lambda r) e^{-\lambda r} .
$$

The graphs of the marginal probability density and the marginal cumulative distribution functions of the polar exponential distribution dependent on $\mathrm{R}$ with parameter $\lambda=2$ are shown in the Figure 5 .

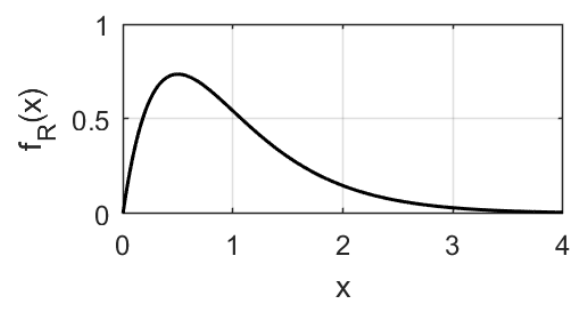

(a)

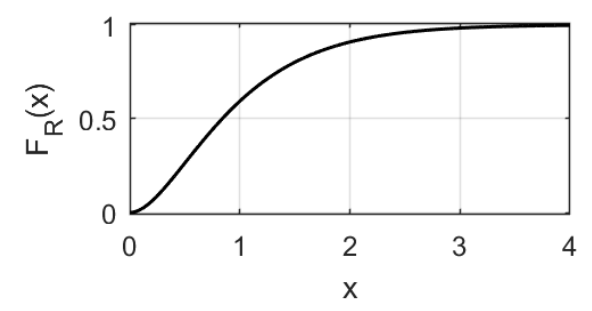

(b)

Figure 5. The polar exponential distribution dependent on $R$ with parameter $\lambda=2 ;(a)$ the marginal probability density function $(b)$ the marginal cumulative distribution function

The marginal probability density function dependent on the $\Theta$ of the polar exponential distribution is calculated by using the Equation (17) as follows [12]

$$
f_{\Theta}(\theta ; \lambda)=\int_{0}^{\infty} \frac{\lambda^{2} e^{-\lambda r}}{2 \pi} r d r
$$

The marginal probability density function dependent on the $\Theta$ of the polar uniform distribution is obtained by calculating integral with bounds as the following Equation (26)

$f_{\Theta}(\theta ; \lambda)=\frac{1}{2 \pi}$. 
If the integral of Equation (26) with bounds $[0, \theta]$ dependent on the $\theta$ is calculated, the Equation (27) is obtained

$$
F_{\Theta}(\theta ; \lambda)=\int_{0}^{\theta} \frac{1}{2 \pi} d \phi
$$

Thus, the marginal cumulative distribution function of the polar exponential distribution dependent on $\Theta$ is found as follows

$$
F_{\Theta}(\theta ; \lambda)=\frac{\theta}{2 \pi}
$$

The graphs of the marginal probability density and the marginal cumulative distribution functions of the polar exponential distribution with parameter $\lambda=2$ dependent on $\Theta$ which are the same as that of the polar uniform distribution, are shown in the Figure 3.

\subsection{Polar Normal Distribution}

When the angular change is taken constant, the probability density function of normal distribution in polar coordinates is calculated as follows [12]

$f_{\Theta, \mathrm{R}}(\theta, r ; \sigma)=\frac{1}{2 \pi \sigma^{2}} e^{-\frac{r^{2}}{2 \sigma^{2}}}$

The graph of this probability density function with parameter $\sigma=1$ is given in Figure 6(a). The distribution function dependent on the variable $r$ is defined in the Equation (30),

$$
F_{\Theta, \mathrm{R}}(\theta, r ; \sigma)=\int_{0}^{\theta} \int_{0}^{r} f_{\Theta, \mathrm{R}}(\phi, \rho ; \sigma) d \rho d \phi,
$$

and if the integral of this function is calculated, the Equation (31) is obtained

$F_{\Theta, \mathrm{R}}(\theta, r ; \sigma)=\frac{\theta}{2 \pi} \int_{0}^{r} \frac{e^{-\frac{\rho^{2}}{2 \sigma^{2}}}}{\sigma^{2}} \rho d \rho$

The cumulative distribution function in the Equation (32) is obtained by calculating integral with bounds

$F_{\Theta, \mathrm{R}}(\theta, r ; \sigma)=\frac{\theta}{2 \pi}\left[1-e^{-\frac{r^{2}}{2 \sigma^{2}}}\right]$

The representations of the probability density and the cumulative distribution functions of the polar normal distribution with parameter $\sigma=1$ are shown in the Figure 6.

The marginal probability density function dependent on the $R$ of the polar normal distribution is calculated by using the Equation (29) as follows [12]

$f_{R}(r ; \sigma)=\int_{0}^{2 \pi} \frac{1}{2 \pi \sigma^{2}} e^{-\frac{r^{2}}{2 \sigma^{2}}} r d \phi$ 


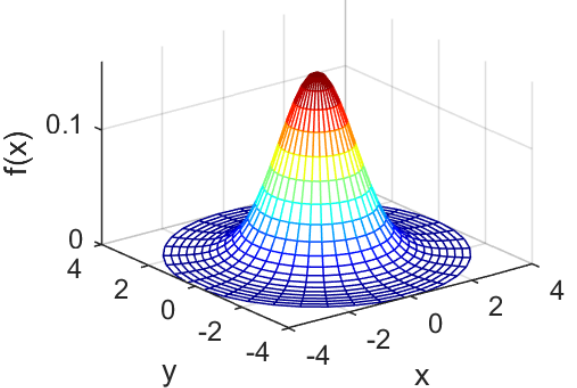

(a)

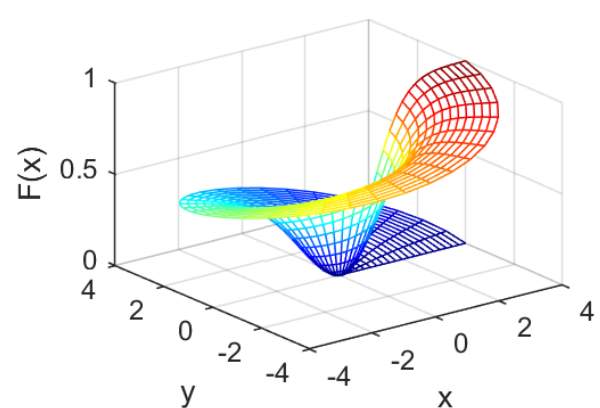

(b)

Figure 6. The polar normal distribution with parameter $\sigma=1$; (a) the probability density function (b) the cumulative distribution function

The probability density function of the polar normal distribution is obtained by calculating integral with bounds as follows

$$
f_{R}(r ; \sigma)=\frac{r}{\sigma^{2}} e^{-\frac{r^{2}}{2 \sigma^{2}}} .
$$

The graph of the marginal probability density function dependent on the $R$ of the polar normal distribution with parameter $\sigma=1$ is given in the Figure 7 (a). If the integral of Equation (34) with bounds [0,r] dependent on the $r$ is calculated, the Equation (35) is obtained

$$
F_{\mathrm{R}}(r ; \sigma)=\int_{0}^{r} \frac{\rho}{\sigma^{2}} e^{-\frac{\rho^{2}}{2 \sigma^{2}}} d \rho .
$$

Thus, the marginal cumulative distribution function of the polar normal distribution dependent on $R$ is found as follows

$$
F_{R}(r ; \sigma)=1-e^{-\frac{r^{2}}{2 \sigma^{2}}}
$$

The graphs of the marginal probability density and the marginal cumulative distribution functions of the polar normal distribution dependent on $\mathrm{R}$ with parameter $\sigma=1$ are shown in the Figure 7 .

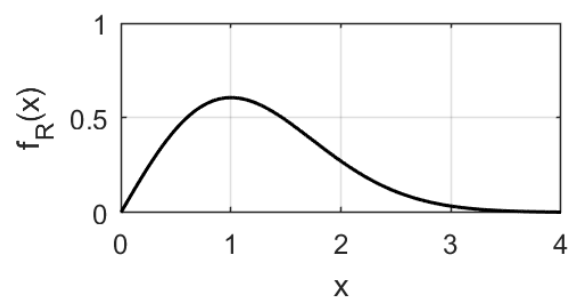

(a)

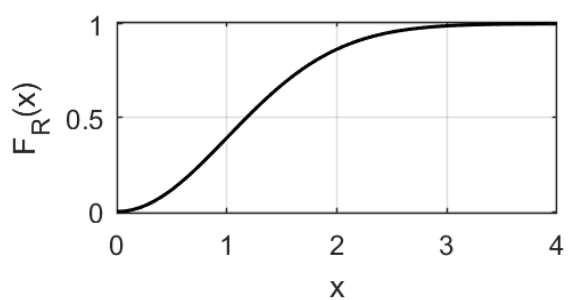

(b)

Figure 7. The polar normal distribution dependent on $R$ with parameter $\sigma=1$; (a) the marginal probability density function (b) the marginal cumulative distribution function

The marginal probability density function dependent on the $\Theta$ of the polar normal distribution is calculated by using the Equation (29) as follows [12]

$$
f_{\Theta}(\theta ; \sigma)=\int_{0}^{\infty} \frac{1}{2 \pi \sigma^{2}} e^{-\frac{r^{2}}{2 \sigma^{2}}} r d r .
$$

The probability density function dependent on the $\Theta$ of the polar normal distribution is obtained by calculating integral with bounds as the following Equation (38) 
$f_{\Theta}(\theta ; \sigma)=\frac{1}{2 \pi}$.

If the integral of Equation (38) with bounds $[0, \theta]$ dependent on the $\theta$ is calculated, the Equation (39) is obtained

$$
F_{\Theta}(\theta ; \sigma)=\int_{0}^{\theta} \frac{1}{2 \pi} d \phi
$$

Thus, the marginal cumulative distribution function of the polar normal distribution dependent on $\Theta$ is found as follows

$$
F_{\Theta}(\theta ; \sigma)=\frac{\theta}{2 \pi}
$$

The graphs of the marginal probability density and the marginal cumulative distribution functions of the polar normal distribution with parameter $\sigma=1$ dependent on $\Theta$ which are the same as that of the polar uniform distribution, are shown in the Figure 3.

\subsection{Polar Triangular Distribution}

The peak of the triangular distribution is in an arbitrary position. Since the peak of the distribution is selected as a starting point in polar coordinates, the probability density function of a special form of triangular distribution is defined as follows [12]

$f_{\Theta, \mathrm{R}}(\theta, r ; b)=\frac{3}{\pi b^{2}}\left(1-\frac{r}{b}\right)$.

In this function, parameter $b$ gives the position where the distribution endpoint. The graph of this probability density function with parameter $b=2$ is given in Figure 8(a). The distribution function dependent on the variable $r$ is defined in the Equation (42),

$$
F_{\Theta, \mathrm{R}}(\theta, r ; b)=\int_{0}^{\theta} \int_{0}^{r} f_{\Theta, \mathrm{R}}(\phi, \rho ; \sigma) d \rho d \phi,
$$

and if the integral of this function is calculated, the Equation (43) is obtained

$$
F_{\Theta, \mathrm{R}}(\theta, r ; b)=\theta \int_{0}^{r} \frac{3}{\pi b^{2}}\left(1-\frac{\rho}{b}\right) \rho d \rho
$$

The cumulative distribution function in the Equation (44) is obtained by calculating integral with bounds

$$
F_{\Theta, \mathrm{R}}(\theta, r ; b)=\frac{\theta}{2 \pi}\left[\frac{r^{2}}{b^{3}}(3 b-2 r)\right]
$$

The representations of the probability density and the cumulative distribution functions of the polar triangular distribution with parameter $b=2$ are shown in the Figure 8.

The marginal probability density function dependent on the $R$ of the polar triangular distribution is calculated by using the Equation (41) as follows [12]

$f_{R}(r ; b)=\int_{0}^{2 \pi} \frac{3}{\pi b^{2}}\left(1-\frac{r}{b}\right) r d \phi$. 


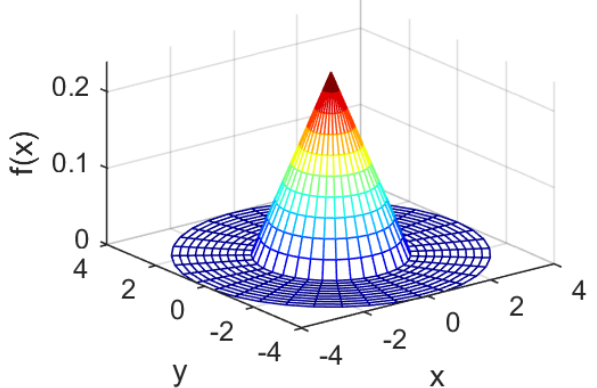

(a)

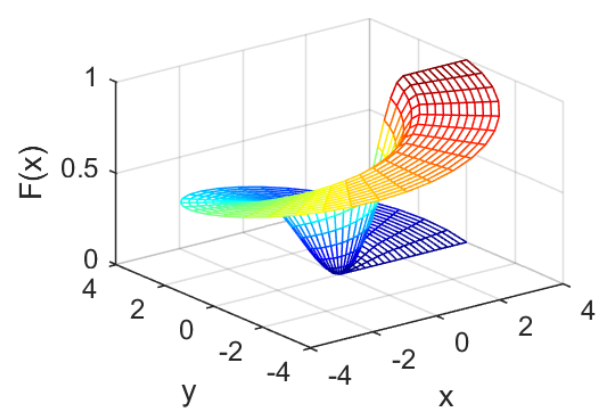

(b)

Figure 8. The polar triangular distribution with parameter $b=2$; (a) the probability density function (b) the cumulative distribution function

The probability density function of the polar triangular distribution is obtained by calculating integral with bounds as follows

$$
f_{R}(r ; b)=\frac{6 r}{b^{2}}\left(1-\frac{r}{b}\right)
$$

The graph of the marginal probability density function dependent on the $R$ of the polar triangular distribution with parameter $b=2$ is given in the Figure 9(a). If the integral of Equation (46) with bounds $[0, r]$ dependent on the $r$ is calculated, the Equation (47) is obtained

$$
F_{\mathrm{R}}(r ; b)=\int_{0}^{r} \frac{6 \rho}{b^{2}}\left(1-\frac{\rho}{b}\right) d \rho
$$

Thus, the marginal cumulative distribution function of the polar triangular distribution dependent on $\mathrm{R}$ is found as follows [12]

$$
F_{R}(r ; b)=\frac{r^{2}}{b^{3}}(3 b-2 r)
$$

The graphs of the marginal probability density and the marginal cumulative distribution functions of the polar triangular distribution dependent on $R$ with parameter $b=2$ are shown in the Figure 9.

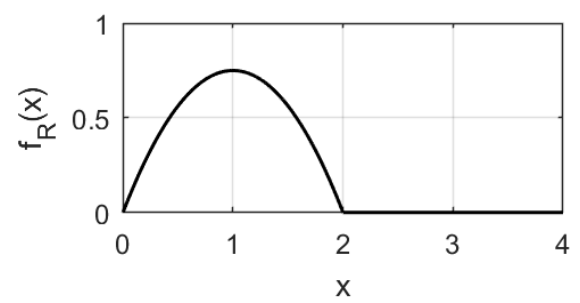

(a)

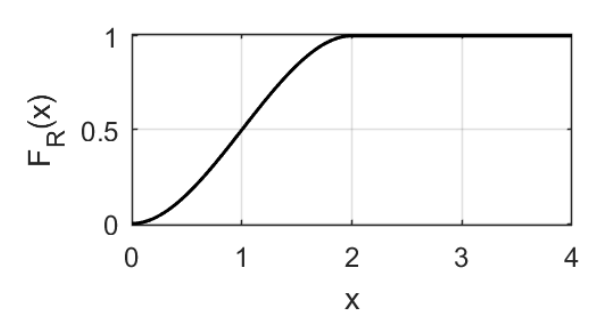

(b)

Figure 9. The polar triangular distribution dependent on $R$ with parameter $b=2 ;(a)$ the marginal probability density function $(b)$ the marginal cumulative distribution function

The marginal probability density function dependent on the $\Theta$ of the polar triangular distribution is calculated by using the Equation (41) as follows [12]

$$
f_{\Theta}(\theta ; b)=\int_{0}^{b} \frac{3}{\pi b^{2}}\left(1-\frac{r}{b}\right) r d r
$$

The probability density function dependent on the $\Theta$ of the polar triangular distribution is obtained by calculating integral with bounds as the following Equation (50)

$$
f_{\Theta}(\theta ; b)=\frac{1}{2 \pi} .
$$


If the integral of Equation (41) with bounds $[0, \theta]$ dependent on the $\theta$ is calculated, the Equation (51) is obtained

$F_{\Theta}(\theta ; b)=\int_{0}^{\theta} \frac{1}{2 \pi} d \phi$

Thus, the marginal cumulative distribution function of the polar triangular distribution dependent on $\Theta$ is found as follows

$F_{\Theta}(\theta ; b)=\frac{\theta}{2 \pi}$.

The graphs of the marginal probability density and the marginal cumulative distribution functions of the polar triangular distribution dependent on $\Theta$ with parameter $b=2$ which are the same as that of the polar uniform distribution, are shown in the Figure 3.

\section{HISTOGRAM IN POLAR COORDINATES}

The histogram shows the frequency of the data. The equal interval is used in univariate variables and the equal area is used in bivariate variables for fitting it to the probability density function. Therefore, while equal areas are used in cartesian coordinates, it is necessary to use equal area slices in polar coordinates for the histogram representation of the bivariate data. Therefore, the angular change is taken in equal interval when calculating the polar histogram. Accordingly, if the whole circle is taken as $2 \pi$ and it is divided into $C_{\theta}$ slices, the interval of each slice can be found as follows

$\Delta \psi=\frac{2 \pi}{C_{\theta}}$.

The boundaries of all slices according to this interval are calculated as follows

$\psi_{j}=j \cdot \Delta \psi, \quad j=0,1,2, \ldots, C_{\theta}$.

The graphical representation for $\Delta \psi=\pi / 6$ is given in the Figure 10 .

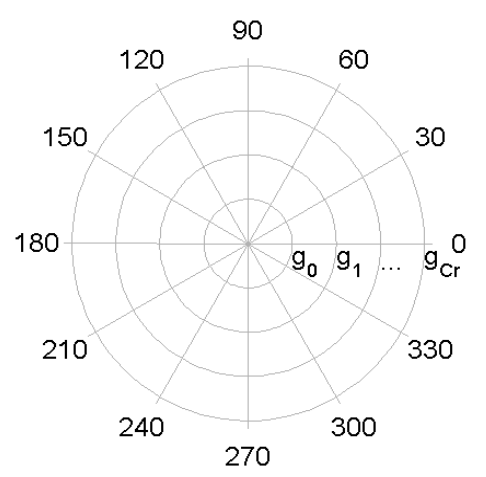

(a)

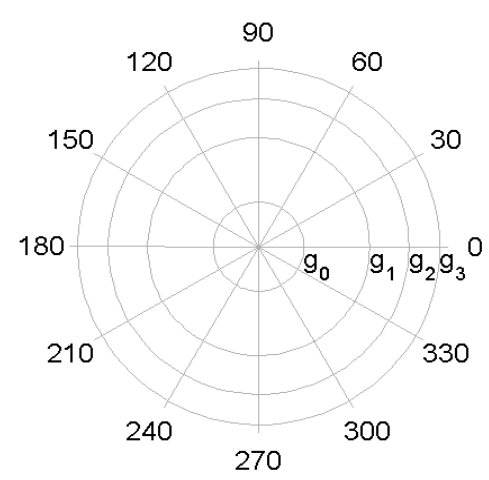

(b)

Figure 10. Representation of the polar grids; (a) Grids with the equal interval radius (b) Grids with the equal slice area

Since the angles has equal interval in polar coordinates, the radii must be selected as a variable for the equal slice areas. In this case, if it is desired to divide the area which is between the inner circle $\left(g_{0}\right)$ and the outer circle $\left(g_{C_{r}}\right)$ into $C_{r}$ circle slice, each round slice area is calculated as follows for each equal circle slices

$A_{k}=\frac{A_{C_{r}}}{C_{r}}, \quad k=1,2, \ldots, C_{r}$

The following equation can be written from this equation 
$A_{k}=\frac{\Delta \psi}{2 \pi} \pi\left(\frac{g_{C_{r}}^{2}-g_{0}^{2}}{C_{r}}\right)$

If the Equation (56) is set for $k=1$, the following equation is obtained

$\frac{\Delta \psi}{2}\left(g_{1}^{2}-g_{0}^{2}\right)=\frac{\Delta \psi}{2}\left(\frac{g_{C_{r}}^{2}-g_{0}^{2}}{C_{r}}\right)$.

If the necessary simplifications are performed, the equality is achieved

$g_{1}^{2}-g_{0}^{2}=\frac{g_{C_{r}}^{2}-g_{0}^{2}}{C_{r}}$.

$g_{1}^{2}$ is calculated in given the Equation (59)

$g_{1}^{2}=\frac{g_{C_{r}}^{2}-g_{0}^{2}}{C_{r}}+g_{0}^{2}$.

Likewise, if the equal area is used for the radii of the slices in the second circle, the Equation (60) can be written

$$
g_{2}^{2}-g_{1}^{2}=\frac{g_{C_{r}}^{2}-g_{0}^{2}}{C_{r}}
$$

$g_{2}^{2}$ is calculated in given the Equation (61)

$g_{2}^{2}=\frac{g_{C_{r}}^{2}-g_{0}^{2}}{C_{r}}+g_{1}^{2}$

The Equation (61) becomes the following equation if the Equation (59) is used instead of $g_{1}^{2}$ in this equation

$g_{2}^{2}=2 \frac{g_{C_{r}}^{2}-g_{0}^{2}}{C_{r}}+g_{0}^{2}$

The radius of the third circle slices is calculated as follows by continuing the process

$g_{3}^{2}=\frac{g_{C_{r}}^{2}-g_{0}^{2}}{C_{r}}+g_{2}^{2}$

The following equation is obtained if the Equation (62) is used instead of $g_{2}^{2}$

$g_{3}^{2}=3 \frac{g_{C_{r}}^{2}-g_{0}^{2}}{C_{r}}+g_{0}^{2}$

If this equality is generalized, the upper radius of $k^{\text {th }}$ the circle slice is obtained by using the Equation (65)

$g_{k}^{2}=k \frac{g_{C_{r}}^{2}-g_{0}^{2}}{C_{r}}+g_{0}^{2}, \quad k=0,1,2, \ldots, c$.

\section{RANDOM NUMBER GENERATION FROM POLAR DISTRIBUTIONS}


Since the marginal distribution functions of the variables $(\Theta$ and $R)$ of the polar distribution function defined in the previous sections satisfy the condition in Equation (66), these two variables are independent of each other

$F_{\Theta, \mathrm{R}}(\theta, r)=F_{\Theta}(\theta) F_{R}(r)$

The marginal distribution functions of two variables are used in order to generate random numbers from the bivariate polar distribution function. In this study, the two most common methods are used to generate random numbers from marginal distribution functions. These methods are the rejection and the inversion methods. Although the inverse method is the most robust method, the rejection method sometimes is used because the inverse of some distributions cannot be calculated [13]. In this study, random numbers are generated from the polar uniform distribution, the polar exponential distribution, the polar normal distribution and the polar triangular distribution.

\subsection{Random Number Generation from Polar Uniform Distribution}

If the Equation (12) equals to a random variable generated from the uniform distribution in the range of $[0,1)$ such as $U$ to generate a random number from the polar uniform distribution, the following equation is obtained

$\frac{R^{2}-a^{2}}{b^{2}-a^{2}}=U$

Therefore, $R$ is calculated in given the Equation (68)

$R=\sqrt{a^{2}+U\left(b^{2}-a^{2}\right)}$.

By means of this equation, the value of the $R$ component for the polar uniform distribution is generated randomly. If the Equation (16) equals to a random variable generated from the uniform distribution in the range of $[0,1)$ such as $U$ for the $\Theta$ component of the polar uniform distribution, the following equation is obtained

$\frac{\Theta}{2 \pi}=U$

$\Theta$ is calculated in given the Equation (70)

$\Theta=2 \pi U$

Using this equation, the value of the $\Theta$ component can be generated randomly.

As an example, 10.000 random numbers are generated from the polar uniform distribution with the parameters $a=1$ and $b=2$, and their polar histogram with equal area is shown in Figure 11(a) and the polar histogram with equal interval is given in Figure 11(b).

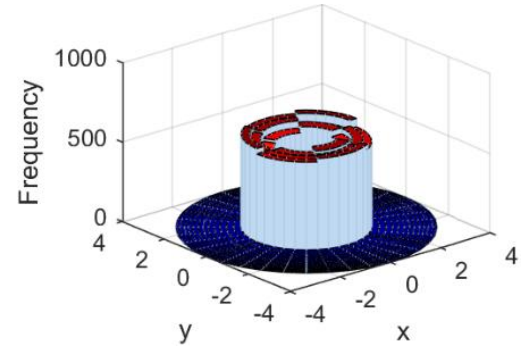

(a)

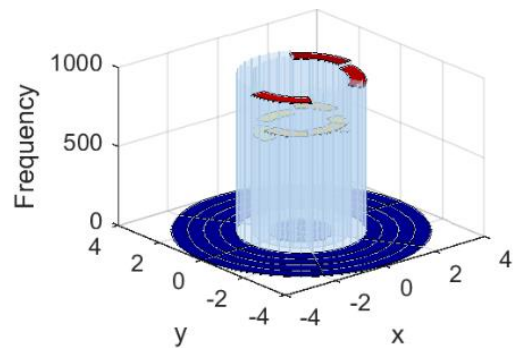

(b) 
Figure 11. Representation of the polar histogram for 10.000 random numbers generated from the polar uniform distribution with the parameters $a=1$ and $b=2$; $(a)$ the equal area (b) the equal interval

\subsection{Random Number Generation from Polar Exponential Distribution}

If the Equation (24) equals to a random variable generated from the uniform distribution in the range of $[0,1)$ such as $U$ to generate a random number from the polar exponential distribution, the following equation is obtained

$1-(1+\lambda R) e^{-\lambda R}=U$

Since the $R$ variable cannot be calculated analytically in this equation, random number generation for the $R$ component from this polar distribution is performed with the help of the rejection method. The auxiliary function selected according to the rejection method is given as follows

$g(r)=\lambda e^{-\lambda r}$

Equation (73) can be used to generate random numbers from this auxiliary function

$V=-\frac{1}{\lambda} \log (1-U)$

It is necessary to find the smallest $\mathrm{c}$ value which satisfies the following condition with the help of the Equation (72)

$\frac{f(r)}{g(r)} \leq c$

Hence, Equation (75) is obtained by using the Equation (22) and the Equation (72)

$\frac{\lambda^{2} r e^{-\lambda r}}{\lambda e^{-\lambda r}} \leq c$

Lastly, the value of $\mathrm{c}$ is calculated as follows

$c \geq \lambda r$.

Algorithm 1. Random number generation by the rejection method

Step 1. Generate the $V$ random value from the $g(r)$ distribution function,

Step 2. Generate random number $(U)$ from uniform distribution with the interval of $[0,1)$,

Step 3. If $U \leq \frac{g(V)}{c . f(V)}, R=V$; Else go to Step 1 .

By means of the Algorithm 1, the value of the $R$ component for the polar exponential distribution is generated randomly. If the Equation (28) equals to a random variable generated from the uniform distribution in the range of $[0,1)$ such as $U$ for the $\Theta$ component of the polar exponential distribution, the value of the $\Theta$ component can be generated randomly as Equation (70).

As an example, 10.000 random numbers are generated from the polar exponential distribution with the parameter $\lambda=2$ and their polar histogram with equal area is shown in Figure 12(a) and the polar histogram with equal interval is given in Figure 12(b). 


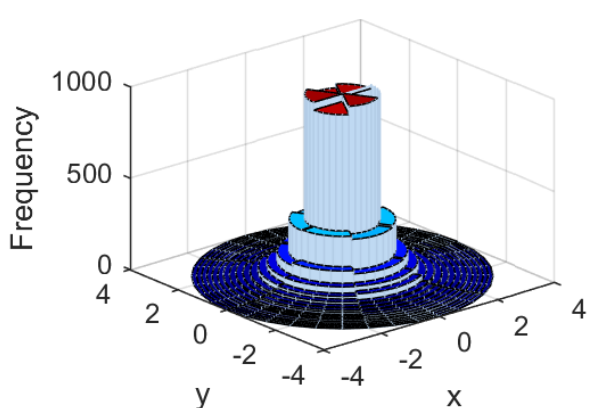

(a)

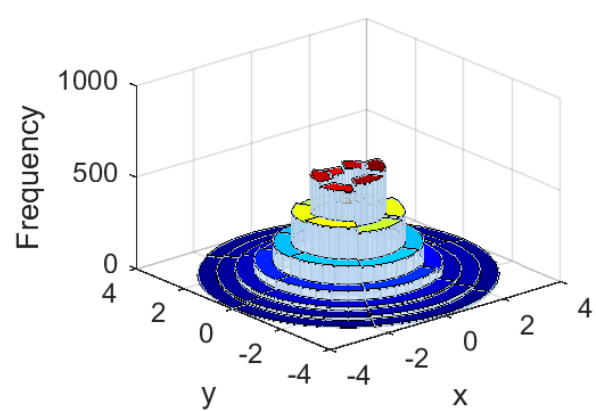

(b)

Figure 12. Representation of the polar histogram for 10.000 random numbers generated from the polar exponential distribution with the parameter $\lambda=2 ;(a)$ the equal area $(b)$ the equal interval

\subsection{Random Number Generation from Polar Normal Distribution}

If the Equation (36) equals to a random variable generated from the uniform distribution in the range of $[0,1)$ such as $U$ to generate a random number from the polar normal distribution, the following equation is obtained

$$
1-e^{-\frac{R^{2}}{2 \sigma^{2}}}=U
$$

Therefore, $R$ is calculated in given the Equation (78)

$$
R=\sigma \sqrt{-2 \log (1-U)} .
$$

By means of this equation, the value of the $R$ component for the polar normal distribution is generated randomly. If the Equation (40) equals to a random variable generated from the uniform distribution in the range of $[0,1)$ such as $U$ for the $\Theta$ component of the polar normal distribution, the value of the $\Theta$ component can be generated randomly as Equation (70).

As an example, 10.000 random numbers are generated from the polar normal distribution with the parameter $\sigma=1$ and their polar histogram with equal area is shown in Figure 13(a) and the polar histogram with equal interval is given in Figure 13(b).

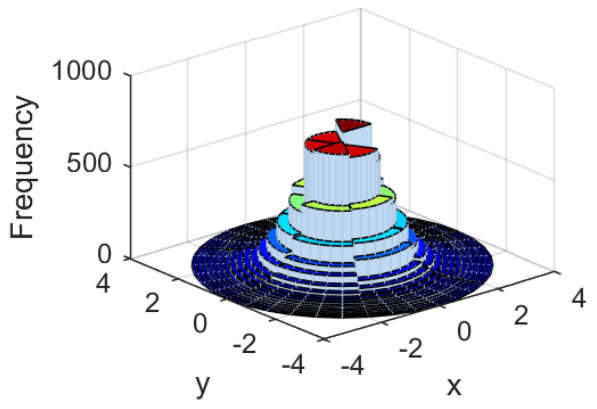

(a)

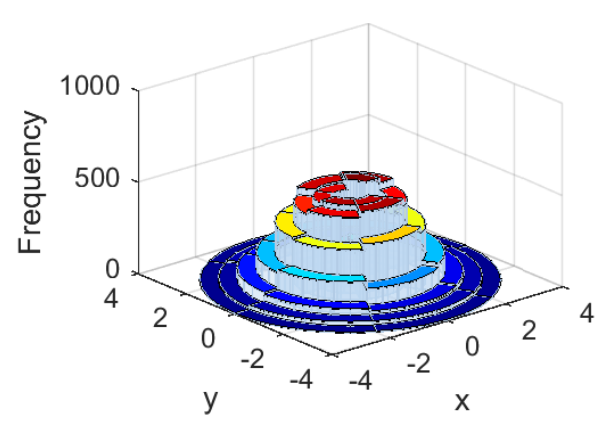

(b)

Figure 13. Representation of the polar histogram for 10.000 random numbers generated from the polar normal distribution with the parameter $\sigma=1 ;(a)$ the equal area $(b)$ the equal interval

\subsection{Random Number Generation from Polar Triangular Distribution}

If the Equation (48) equals to a random variable generated from the uniform distribution in the range of $[0,1)$ such as $U$ to generate a random number from the polar triangular distribution, the following equation is obtained 
$\frac{R^{2}}{b^{3}}(3 b-2 R)=U$

Since the $R$ variable cannot be calculated easily in this equation, random number generation for the $R$ component from this polar distribution is performed with the help of the rejection method. The auxiliary function selected according to the rejection method is given as follows

$g(r)=\frac{2}{b}\left(1-\frac{r}{b}\right)$

Equation (81) can be used to generate random numbers from this auxiliary function

$V=b-b \sqrt{1-U}$.

It is necessary to find the smallest $\mathrm{c}$ value which satisfies the following condition with the help of the Equation (80)

$\frac{f(r)}{g(r)} \leq c$

Hence, Equation (83) is obtained by using the Equation (46) and the Equation (80)

$\frac{\frac{6 r}{b^{2}}\left(1-\frac{r}{b}\right)}{\frac{2}{b}\left(1-\frac{r}{b}\right)} \leq c$

Lastly, the value of $\mathrm{c}$ is calculated as follows

$c \geq \frac{3 r}{b}$

By means of the Algorithm 1, the value of the $R$ component for the polar triangular distribution is generated randomly. If the Equation (52) equals to a random variable generated from the uniform distribution in the range of $[0,1)$ such as $U$ for the $\Theta$ component of the polar triangular distribution, the value of the $\Theta$ component can be generated randomly as Equation (70).

As an example, 10.000 random numbers are generated from the polar triangular distribution with the parameter $b=2$ and their polar histogram with equal area is shown in Figure 14(a) and the polar histogram with equal interval is given in Figure 14(b).

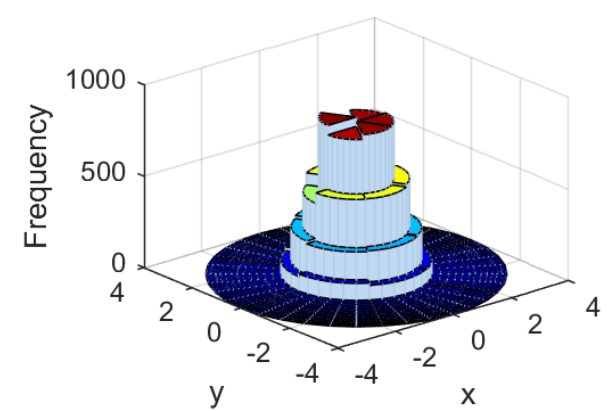

(a)

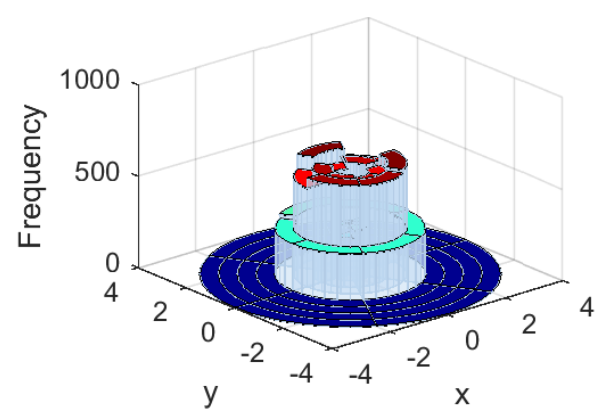

(b)

Figure 14. Representation of the polar histogram for 10.000 random numbers generated from the polar triangular distribution with the parameter $b=2 ;(a)$ the equal area $(b)$ the equal interval

\section{CHI-SQUARE GOODNESS OF FIT TEST IN POLAR COORDINATES}

In this study, chi-square goodness of fit test is used for the defined distributions in polar coordinates. Because it is one of the most common methods of goodness of fit tests. In the tests to be performed with 
the use of marginal distribution functions in the goodness of fit tests, the acceptability of the $H_{0}$ hypothesis at the same time of the results of two separate tests to be performed according to the spatial and angular variables are calculated according to $(1-\alpha)^{2}$ [14]. In this case, since the margin of error $\left(\hat{\alpha}=1-(1-\alpha)^{2}\right)$ increases, undesirable results will be obtained for goodness of fit test. As a temporary solution to the problem, the goodness of fit of the spatial variable is tested by assuming that the angular variable has uniform distribution. The chi-square goodness of fit test is performed both in a bivariate sliced area and using marginal functions.

\subsection{Sliced Bivariate Chi-Square Test}

Let the sample values $(\theta, r)=\left\{\left(\theta_{i}, r_{i}\right), i=1,2, \ldots, n\right\}$ are obtained from an independent and continuous identical distribution $F(\theta, r)$. In this test, the null hypothesis $\left(H_{0}\right)$ and the alternative hypothesis $\left(H_{1}\right)$ are based on two hypotheses as follows

$H_{0}$ : Observation values $F_{0}(\theta, r)$ have $F_{0}(\theta, r)$ distribution $\left(F(\theta, r)=F_{0}(\theta, r)\right)$,

$H_{0}$ : Observation values $F_{0}(\theta, r)$ have not $F_{0}(\theta, r)$ distribution $\left(F(\theta, r) \neq F_{0}(\theta, r)\right)$.

Also, $F_{0}(\theta, r)$ is the distribution function that assumes the observation values have. In addition, a significance level $(\alpha)$ is determined for the tests. First, the number of classes and the areas of classes must be determined in order to perform the chi-square test on continuous functions. Although there are various studies in the literature in determining the number of classes $\left(C_{\theta}, C_{r}\right)$, it is often left to the researcher's choice. Angular and spatial class boundaries are given in the Equation (54) and the Equation (65).

According to these equations, the number of observations (observed frequencies) in per class area is calculated as follows

$$
o_{j k}=\sum_{i=1}^{n}\left\{\begin{array}{c}
1, \quad\left(\psi_{j-1} \leq \theta_{i}<\psi_{j}\right) \wedge\left(g_{k-1} \leq r_{i}<g_{k}\right) \\
0, \\
\text { otherwise } \quad\left(j=1,2, \ldots, C_{\theta} ; k=1,2, \ldots, C_{r}\right) .
\end{array}\right.
$$

Also, the expected frequencies are calculated as given the Equation (86)

$$
\begin{aligned}
E_{j k}=n\left[F_{0}\left(\psi_{j}, g_{k}\right)-F_{0}\left(\psi_{j}, g_{k-1}\right)-F_{0}\left(\psi_{j-1}, g_{k}\right)+F_{0}\left(\psi_{j-1}, g_{k-1}\right)\right], & \left(j=1,2, \ldots, C_{\theta} ; k=1,2, \ldots, C_{r}\right) .
\end{aligned}
$$

The chi-square value is calculated in given the Equation (87) with the help of the expected and the observed frequencies

$$
\chi_{h}^{2}=\sum_{j=1}^{C_{\theta}} \sum_{k=1}^{C_{r}} \frac{\left(o_{j k}-E_{j k}\right)^{2}}{E_{j k}}
$$

In the next step, $\chi_{v, 1-\alpha}^{2}$ value which gives the confidence level $(1-\alpha)$ from the chi-square distribution should be found according to the given significance level. It is found by using the chi-square table [15]. Consequently, if the following inequality is true, the null hypothesis $\left(H_{0}\right)$ is accepted. Otherwise, the null hypothesis is rejected

$$
\chi_{h}^{2}<\chi_{v, 1-\alpha}^{2} .
$$

In there, $v$ is the degree of freedom and is found with the equation $v=C_{\theta} C_{r}-1$. 


\subsection{Marginal Chi-Square Test}

When the goodness of fit test is performed according to the marginal distribution functions, the chi-square test is performed by assuming that the angular variable has uniform distribution. Let the sample values $r=\left\{r_{i}, \quad i=1,2, \ldots, n\right\}$ are obtained from an independent and continuous identical distribution $F_{R}(r)$ for the univariate polar chi-square test.

In this test, the null hypothesis $\left(H_{0}\right)$ and the alternative hypothesis $\left(H_{1}\right)$ are based on two hypotheses as follows

$$
\begin{aligned}
& H_{0}: \text { Observation values have the } F_{0}(r) \text { distribution }\left(F_{R}(r)=F_{0}(r)\right), \\
& H_{0}: \text { Observation values have not the } F_{0}(r) \text { distribution }\left(F_{R}(r) \neq F_{0}(r)\right) \text {. }
\end{aligned}
$$

Also, $F_{0}(r)$ is the distribution function that assumes the observation values have. In addition, a significance level $(\alpha)$ is determined for the tests. Although there are various studies in the literature in determining the number of classes $\left(C_{r}\right)$, it is often left to the researcher's choice. Furthermore, the class width is calculated with two methods. The first method is to divide the difference between the maximum value and the smallest value into equal intervals, and the second method is to divide the arbitrary upper and lower bounds into equal intervals. In this study, the class width is calculated as in the Equation (89)

$$
\Delta g=\frac{r_{n}-r_{1}}{C_{r}}
$$

In there, $r_{1}$ represents the smallest value in the data and $r_{n}$ represents the largest value in the data. The $j$. upper bound of the class is calculated as follows

$$
g_{j}=r_{1}+(j-1) . \Delta g, \quad j=1,2, \ldots, C_{r} .
$$

The number of observations (observed frequencies) in per class interval is calculated as in Equation (90)

$$
O_{j}=\sum_{i=1}^{n}\left\{\begin{array}{cc}
1, & g_{j-1} \leq r_{i}<g_{j} \\
0, & \text { otherwise }
\end{array}, \quad j=1,2, \ldots, C_{r} .\right.
$$

And, the expected frequencies are calculated as following equation

$$
E_{j}=n\left[F_{0}\left(g_{j}\right)-F_{0}\left(g_{j-1}\right)\right], \quad j=1,2, \ldots, C_{r} .
$$

$F_{0}$ represents the distribution function of the examined probability density function. The chi-square value is calculated in given the Equation (93) with the help of the expected and the observed frequencies

$$
\chi_{h}^{2}=\sum_{j=1}^{C_{r}} \frac{\left(o_{j}-E_{j}\right)^{2}}{E_{j}}
$$

The comparison of the calculated chi-square statistic $\chi_{h}^{2}$ with the chi-square table value at the confidence level $(1-\alpha)$ with the degree of freedom $\left(v=C_{r}-1\right)$ is performed as follows

$$
\chi_{h}^{2}<\chi_{v, 1-\alpha}^{2}
$$

If the test statistic $\left(\chi_{h}^{2}\right)$ is less than the table value $\left(\chi_{v, 1-\alpha}^{2}\right)$, the null hypothesis $\left(H_{0}\right)$ is accepted. Otherwise, the null hypothesis is rejected. 


\section{EXPERIMENTAL RESULTS}

In this section, four different distribution functions are selected to evaluate the success of the chi-square goodness of fit test for polar distribution functions. The success of the chi-square goodness of fit test is performed at the $\mathrm{n}=10,30,50,100,200,300$ sample sizes for these distributions. Two-dimensional chisquare $\left(2 D-\chi^{2}\right)$ and one-dimensional chi-square $\left(1 D-\chi^{2}\right)$ tests are used for these samples. For each sample size, this process is repeated 10,000 . The success rates of the generated random numbers from the polar uniform distribution with the parameters $a=1$ and $b=2$ are given in Table 1 . All values in Table 1 are around $95 \%$.

Table 1. Simulation results for the marginal cumulative distribution of polar uniform distribution

\begin{tabular}{lll}
\hline $\boldsymbol{n}$ & $\mathbf{2 D}-\boldsymbol{\chi}^{\mathbf{2}}(\%)$ & $\mathbf{1 D}-\boldsymbol{\chi}^{\mathbf{2}}(\%)$ \\
\hline 50 & 94.14 & 94.79 \\
100 & 94.43 & 94.84 \\
200 & 95.12 & 95.20 \\
300 & 95.10 & 95.05 \\
500 & 95.13 & 94.73 \\
\hline
\end{tabular}

The success rates of the generated random numbers from the polar exponential distribution with the parameter $\lambda=2$ are given in Table 2 . While the success rates of the one-dimensional chi-square $\left(1 D-\chi^{2}\right)$ test are around at $95 \%$, the success rates of the two-dimensional chi-square $\left(2 D-\chi^{2}\right)$ test are less than at $95 \%$ in Table 2. However, as the sample size increases, these success rates approach $95 \%$. Because the expected frequency value in per class is less than 5. In this case, reclassification can be made by combining classes which have the expected frequency less than 5 and neighbor classes. Another approach is to classify the expected value of each class to be equal.

Table 2. Simulation results for the marginal cumulative distribution of polar exponential distribution

\begin{tabular}{lll}
\hline $\boldsymbol{n}$ & $\mathbf{2 D}-\boldsymbol{\chi}^{\mathbf{2}(\%)}$ & $\mathbf{1 D}-\chi^{\mathbf{2}}(\%)$ \\
\hline 50 & 89.54 & 94.67 \\
100 & 91.42 & 95.04 \\
200 & 92.60 & 94.97 \\
300 & 93.16 & 95.09 \\
500 & 93.85 & 95.06 \\
\hline
\end{tabular}

The success rates of the generated random numbers from the polar normal distribution with the parameter $\sigma=1$ are given in Table 3 . The success rates of the one-dimensional chi-square $\left(1 D-\chi^{2}\right)$ test are around at $95 \%$. On the other hand, as the sample size increases, the success rates of the two-dimensional chi-square $\left(2 D-\chi^{2}\right)$ test approach around at $95 \%$ as in Table 3.

Table 3. Simulation results for the marginal cumulative distribution of polar normal distribution

\begin{tabular}{lll}
\hline $\boldsymbol{n}$ & $\mathbf{2 D}-\boldsymbol{\chi}^{\mathbf{2}}(\%)$ & $\mathbf{1 D}-\boldsymbol{\chi}^{\mathbf{2}}(\%)$ \\
\hline 50 & 91.98 & 94.95 \\
100 & 92.65 & 95.38 \\
200 & 93.44 & 95.14 \\
300 & 93.91 & 94.95 \\
500 & 94.24 & 95.03 \\
\hline
\end{tabular}

The success rates of the generated random numbers from the polar triangular distribution with the parameter $b=2$ are given in Table 4 . The results in Table 4 are similar to Table 2 and Table 3 . Also, the success rates of the one-dimensional chi-square $\left(1 D-\chi^{2}\right)$ test are around at $95 \%$. On the other hand, as the sample size increases, the success rates of the two-dimensional chi-square $\left(2 D-\chi^{2}\right)$ test approach around at $95 \%$. 
Table 4. Simulation results for the marginal cumulative distribution of polar triangular distribution

\begin{tabular}{lll}
\hline $\boldsymbol{n}$ & $\mathbf{2 D}-\boldsymbol{\chi}^{\mathbf{2}}(\%)$ & $\mathbf{1 D}-\boldsymbol{\chi}^{\mathbf{2}}(\%)$ \\
\hline 50 & 92.90 & 95.11 \\
100 & 94.04 & 95.04 \\
200 & 94.75 & 95.00 \\
300 & 94.71 & 95.16 \\
500 & 94.56 & 95.26 \\
\hline
\end{tabular}

The marginal goodness of fit tests performs correctly and consistently according to these results for polar distributions. As the sample size increases, the performance of the proposed method increases for twodimensional chi-square test.

\section{REAL-LIFE EXAMPLE}

As a real-life application, the chi-square goodness of fit test was performed in polar coordinates using the angle values and lengths of the branches of the spruce tree. In this data set, the orientations $(\theta)$ and lengths $(r)$ of the branches of the 16 spruce trees at the Kanuni campus of the Karadeniz Technical University were recorded [16]. One of these spruce trees was chosen randomly. The length and angle values of each branch of this tree are given in Table 5.

Table 5. The angles and lengths of the branches of the spruce tree

\begin{tabular}{llllll}
\hline $\begin{array}{l}\text { Branch } \\
\text { No }\end{array}$ & Angle $(\boldsymbol{\theta})$ & Distance $(\boldsymbol{r})$ & $\begin{array}{c}\text { Branch } \\
\text { No }\end{array}$ & Angle $(\boldsymbol{\theta})$ & Distance $(\boldsymbol{r})$ \\
\hline $\mathbf{1}$ & 99 & 35 & $\mathbf{1 1}$ & 325 & 63 \\
$\mathbf{2}$ & 95 & 46 & $\mathbf{1 2}$ & 274 & 141 \\
$\mathbf{3}$ & 91 & 77 & $\mathbf{1 3}$ & 273 & 71 \\
$\mathbf{4}$ & 85 & 139 & $\mathbf{1 4}$ & 269 & 46 \\
$\mathbf{5}$ & 80 & 109 & $\mathbf{1 5}$ & 261 & 38 \\
$\mathbf{6}$ & 50 & 98 & $\mathbf{1 6}$ & 255 & 112 \\
$\mathbf{7}$ & 40 & 112 & $\mathbf{1 7}$ & 205 & 110 \\
$\mathbf{8}$ & 38 & 64 & $\mathbf{1 8}$ & 195 & 42 \\
$\mathbf{9}$ & 29 & 135 & $\mathbf{1 9}$ & 188 & 82 \\
$\mathbf{1 0}$ & 334 & 136 & $\mathbf{2 0}$ & 175 & 81 \\
& & & $\mathbf{2 1}$ & 170 & 149 \\
\hline
\end{tabular}

Whether the data shows polar uniform or polar normal distribution is investigated with the proposed goodness of fit test in polar coordinates. Firstly, the angle values must show a uniform distribution in the range of $[0,2 \pi]$ in order to use the proposed goodness of fit test in polar coordinates. Therefore, the Kolmogorov-Smirnov goodness of fit test is applied to show that whether the angle values have a uniform distribution in the range of $[0,2 \pi]$ or not. Also, the angle values are converted from degrees to radians. The null hypothesis $\left(H_{0}\right)$ and the alternative hypothesis $\left(H_{1}\right)$ are as follows

$H_{0}$ : The angle values have a uniform distribution in the range of $[0,2 \pi]$,

$H_{1}$ :The angle values have not a uniform distribution in the range of $[0,2 \pi]$.

As a result of the Kolmogorov-Smirnov test, since the value of $p=0.6498$ is greater than $\alpha=0.05$, the $H_{0}$ hypothesis is not rejected at the $95 \%$ confidence level. Thereby, the angle values have a uniform distribution in the range of $[0,2 \pi]$.

Firstly, it is tested that whether the spruce tree data has a polar uniform distribution or not. For this test, parameters $[a, b]$ of the uniform distribution need to be estimated. According to the maximum likelihood estimation method, the parameters of the uniform distribution are estimated as $\hat{a}=35$ and $\hat{b}=149$. The null hypothesis $\left(H_{0}\right)$ and the alternative hypothesis $\left(H_{1}\right)$ are as follows 
$H_{0}:$ The spruce tree data has a polar uniform distribution, $H_{1}$ : The spruce tree data has not a polar uniform distribution.

The proposed chi-square goodness of fit test in polar coordinates is carried out according to these values. As a result of the sliced bivariate chi-square test and the marginal chi-square test, $\mathrm{p}$ values are 0 . Since the $\mathrm{p}$ value is less than $\alpha=0.05$, the $H_{0}$ hypothesis is rejected at the $95 \%$ confidence level. The spruce tree data has not a polar uniform distribution at the $95 \%$ confidence level.

Lastly, it is tested that whether the spruce tree data has a polar normal distribution or not. For this test, parameter $(\sigma)$ of the normal distribution need to be estimated. The other parameter $(\mu)$ of the normal distribution does not need. Because there is only one tree. Furthermore, it is accepted as the center and the value of $\mu$ is taken as 0 . According to the maximum likelihood estimation method, the parameter of the normal distribution is estimated as $\hat{\sigma}=36.78$. The null hypothesis $\left(H_{0}\right)$ and the alternative hypothesis $\left(H_{1}\right)$ are as follows.

\section{$H_{0}:$ The spruce tree data has a polar normal distribution. \\ $H_{0}$ : The spruce tree data has not a polar normal distribution.}

The proposed chi-square goodness of fit test in polar coordinates is performed according to this parameter $(\hat{\sigma})$. As a result of the sliced bivariate chi-square test and the marginal chi-square test, $\mathrm{p}$ values are 1 . Since the $\mathrm{p}$ value is greater than $\alpha=0.05$, the $H_{0}$ hypothesis is not rejected at the $95 \%$ confidence level. The spruce tree data has a polar normal distribution at the $95 \%$ confidence level.

\section{CONCLUSION}

In this study, the bivariate distribution function is defined in polar coordinates and some of these statistics are emphasized. While defining bivariate polar distribution functions, it is provided that the variables are independent of each other. And, goodness of fit test is developed according to this condition. The chi-square test can be applied directly to the data obtained from bivariate polar distribution functions. Equivalence of the distributions, which are obtained in cartesian coordinate system, in polar coordinate system is defined. The marginal distribution functions of polar distributions are defined, and random numbers are generated from these distributions in polar coordinates by using the rejection and the inverse methods. In addition, goodness of fit test is applied to these generated random numbers and their performance is tested. The chisquare test is chosen as goodness of fit test in this study. Also, it is used by adapting to polar coordinates. And, it is applied according to the marginal distribution of the spatial variable assuming that the angular variable has uniform distribution.

On the other hand, the parameter estimation is a very important issue in polar coordinates. Polar distributions have two variables such as distance $(r)$ and angle $(\theta)$. In this study, it is assumed that the angle values have a uniform distribution as stated in the title of the study. For this reason, a single variable (distance) was used for parameter estimation of polar uniform and polar normal distributions in the RealLife Example Section. In other words, the distribution parameters from the ordinary (not polar) distributions are estimated by maximum likelihood estimation (mle) method.

Consequently, distribution functions in polar coordinates are defined, random numbers are generated from these distributions and polar goodness test of is performed on these generated random numbers.

\section{CONFLICTS OF INTEREST}

No conflict of interest was declared by the authors.

\section{REFERENCES}

[1] Alford, M.H., MacKinnon J.A., Pinkel R. and Klymak J.M., "Space-time scales of shear in the North Pacific", Journal of Physical Oceanography, 47(10): 2455-2478, (2017). 
[2] Solman, G.J., Kingstone, A., "Balancing energetic and cognitive resources: Memory use during search depends on the orienting effector", Cognition, 132(3): 443-454, (2014).

[3] Schönefeld, J., "Architectures for Embedded Multimodal Sensor Data Fusion Systems in the Robotics -and Airport Traffic Surveillance - Domain", Phd.Thesis, Clausthal University of Technology, Clausthal-Zellerfeld, 28-35 (2015).

[4] Cumming, B.G., DeAngelis, G.C., "The physiology of stereopsis", Annual Review of Neuroscience, 24(1): 203-238, (2001).

[5] Robinson, N.H., Newton, H.M, Allan, J.D., Irwin, M., Hamilton, J.F., Flynn, M., Bower, K.N., Williams, P.I., Mills, G., Reeves, C.E., McFiggans, G., and Coe, H., "Source attribution of Bornean air masses by back trajectory analysis during the OP3 project", Atmospheric Chemistry and Physics, 11(18): 9605-9630, (2011).

[6] Schomaker, L., Bulacu, M., and van Erp, M., "Sparse-parametric writer identification using heterogeneous feature groups", International Conference on Image Processing, Barcelona, Spain, 545-549 (2003).

[7] Fazio, V.S., Komitov, L., Radüge, C., Lagerwall, S.T., and Motschmann, H., "Influence of the flow on the anchoring of nematic liquid crystals on Langmuir-Blodgett monolayers studied by optical second-harmonic generation", The European Physical Journal E, 5(3): 309-315, (2001).

[8] Kesemen, O., Tiryaki, B.K., and Tuncay, U., "A Novel Approximation for Computation Bivariate Distribution Functions in Polygonal Area", 5th International Eurasian Conference on Mathematical Sciences and Applications (IECMSA-2016), Belgrade, 191-191 (2016).

[9] Papoulis, A., Pillai, S.U., Probability, Random Variables, and Stochastic Processes 4th ed., McGraw Hill, New York, (2002).

[10] Ertel, B.R., Reed, H.J., "Angle and time of arrival statistics for circular and elliptical scattering models", IEEE Journal on Selected Areas in Communications, 17(11): 1829-1840, (1999).

[11] Kreyszig, E., Advanced Engineering Mathematics, John Willey \& Sons Inc., New York, (1999).

[12] Pak, A., "Kutupsal Koordinatlarda Ki-Kare ve Kolmogorov-Smirnov Uyum İyiliği Testleri”, MSc. Thesis, Karadeniz Technical University, Trabzon, 19-43 (2017).

[13] Devroye, L., Non-Uniform Random Variate Generation, Springer, New York, (1986).

[14] Marques de Sà, J.P., Applied Statistics using SPSS, STATISTICA, MATLAB and R, Springer, New York, (2007).

[15] O'connor, P., Kleyner, A., Practical Reliability Engineering 5th ed., John Wiley \& Sons, New York, (2012).

[16] Kesemen, O., Tiryaki, B.K., Tezel, Ö., and Özkul, E., "Bitki Dallarının Ortalama Yönelim Sayılarının Belirlenmesinde Yönsel Kümeleme Yaklaşımı", XVIII. International Symposium on Econometrics, Operations Research and Statistics, Trabzon, 364, (2017). 